\title{
Mechanisms of spindle assembly and size control
}

Gabriel Guilloux ${ }^{1}$ and Romain Gibeaux ${ }^{1 *}$

${ }^{1}$ Univ Rennes, CNRS, IGDR (Institute of Genetics and Development of Rennes), UMR 6290, F-35000 Rennes, France

*Correspondence: $\quad$ romain.gibeaux@univ-renes1.fr

$+33(0) 223234676$

\begin{abstract}
The spindle is crucial for cell division by allowing the faithful segregation of replicated chromosomes to daughter cells. Proper segregation is ensured only if microtubules and hundreds of other associated factors interact to assemble this complex structure with the appropriate architecture and size. In this review, we describe the latest view of spindle organization as well as the molecular gradients and mechanisms underlying microtubule nucleation and spindle assembly. We then discuss the overlapping physical and molecular constraints that dictate spindle morphology, concluding with a focus on spindle size regulation.
\end{abstract}

\section{Running title}

Spindle assembly and size control

Keywords

Spindle, Mitosis, Meiosis, Microtubules, Spindle scaling. 


\section{Introduction}

One process essential to life, from organism development and homeostasis to reproduction, is cell division, which allows the faithful segregation of duplicated genetic material from mother to daughter cells. Any dysfunction in this process can potentially lead to an abnormal number of chromosomes in daughter cells, called aneuploidy, and is associated with numerous chromosomal disorders and many pathologies in humans, such as cancers (Chunduri and Storchová, 2019). To ensure the accurate segregation of chromosomes in both meiosis and mitosis, the spindle, a dynamic structure mainly composed of microtubules (MTs), is assembled. This structure provides the necessary forces to align the chromosomes at its center, known as the metaphase plate, and then physically separate the sister chromatids to each daughter cell during anaphase.

Although this MT-based assembly was first described at the end of the $19^{\text {th }}$ century by pioneers in mitosis research, in particular Walther Flemming (Flemming, 1882), the precise mechanisms of spindle assembly and chromosome segregation are still not fully understood. In fact, these questions remain very challenging due to the complexity of this structure which is composed of thousands of MTs and hundreds of proteins (Sauer et al., 2005) or nonprotein factors (Chang et al., 2004; Groen et al., 2011) that can assemble together through various mechanisms and pathways. The substructure of the spindle is in constant flux (Mitchison, 1989; Rogers et al., 2005) due to the dynamic instability of MTs (Mitchison and Kirschner, 1984), which cycle through phases of polymerization and depolymerization through addition and loss of tubulin subunits at their ends, stochastically undergoing transitions between growth and shrinkage (catastrophe) and shrinkage to growth (rescue) (Walker et al., 1988). In addition, a variety of motor proteins translocate MTs within the spindle (Yang et al., 2008; Gatlin and Bloom, 2010). Notably, despite its universal function in eukaryotes, spindle size and morphology vary dramatically across species as well as during the development of an organism (Crowder et al., 2015).

Studies over the past few decades have provided new insight into some of the key conserved mechanisms regulating spindle size and architecture. In this review, we first describe the main pathways responsible for spindle MT nucleation and assembly, focusing on activity gradients and mechanisms of MT amplification. Next, we discuss the physical properties and constraints that influence metaphase spindle size. Finally, we address the role of specific molecular actors that have recently emerged as key regulators of spindle size and morphology 


\section{Spindle microtubule organization}

During interphase, MTs, as part of the cytoskeleton, play a structural role and serve in particular as tracks for vesicles and organelle transport. When the cell transitions into mitosis, the MT network is completely disassembled and spindle MTs are generated de novo (Zhai et al., 1996; Petry, 2016). Although MTs are the main component of spindles, not all MTs are equal in terms of function within the spindle and three main populations can be distinguished: astral MTs, k-fibers, and non-kinetochore MTs (Figure 1).

Astral MTs, found at the spindle poles, were first described as "astral rays" (Inoué and Sato, 2008) that project filaments from the spindle poles toward the plasma membrane. By interacting with molecular factors at the cell cortex, they provide the necessary forces to orient and position the spindle in dividing cells (Pietro et al., 2016). Even though astral MTs were shown to be important for spindle orientation and asymmetric cell division (Siller and Doe, 2009), they are absent or not long enough to extend from pole to cell membrane in cells without centrosomes or in very large embryonic cells, respectively. In such cases, other players, such as for instance acentriolar MT organizing centers or the actin network, function to position the spindle (Moorhouse and Burgess, 2014; Bennabi et al., 2016; Mogessie et al., 2018). Bundles of MTs, called k-fibers, connect the spindle to the chromosomes through a macromolecular complex termed the kinetochore (Cheeseman and Desai, 2008), which assembles at the centromere of each sister chromatid at the onset of mitosis. K-fibers connect sister chromatids to opposite spindle poles and drive the alignment of chromosomes at the metaphase plate. These fibers are the most stable population of MTs in the spindle, are resistant to low temperature-induced depolymerization and possess distinct post-translational modifications of their tubulin subunits (Janke and Bulinski, 2011; Barisic et al., 2015). Of note, it has been shown that the formation of a spindle composed only of k-fibers could be induced using a gentle method of cooling (Rieder, 1981). Interestingly, non-kinetochore MTs linking sister chromatids, called bridging fibers, were recently discovered in HeLa and PtK1 cells (Kajtez et al., 2016). Using laser-cutting, the authors demonstrated that these fibers contribute to the tension between sister kinetochores, and that their thickness defines the forces at the spindle poles and kinetochores. The third category of MTs structuring the spindle is generally termed non-kinetochore MTs, and can be further classified into polar and interpolar MTs. Whereas polar MTs are short and present only within half the spindle, interpolar MTs bind to MTs extending from the opposite pole in an antiparallel manner to form overlapping bundles (Brinkley and Cartwright, 1971). In addition, interpolar MTs have 
been demonstrated to play a key role in the spindle elongation that occurs in late anaphase as MTs grow and slide along each other (Masuda et al., 1990; Scholey et al., 2016).

Within the spindle, these populations are intertwined and cooperate to ensure that the spindle is fully functional. Nonetheless, this complex assembly is only possible thanks to the cooperative and coordinated action of MT molecular motors (Figure 1, insets 1-4). While plus-end-directed antiparallel cross-linking motors, in particular the Kinesin-5 Eg5, sort MTs into antiparallel arrays and maintain the association of interpolar MTs, minus-end-directed cross linking motors, including the Kinesin-14 HSET/XCTK2 and Dynein, act to focus of MTs into spindle poles (Walczak et al., 1998; Walczak and Heald, 2008; Ems-McClung et al., 2020). In addition, CENP-E at kinetochores, and chromokinesins on chromosome arms, walk along stable detyrosinated MTs of k-fibers to move chromosomes and maintain their position at the metaphase plate (Barisic et al., 2015). Strikingly, the relative proportions of MTs within each subpopulation varies among cell type and species, but the fact that all three populations are present in most species suggests a strong evolutionary constraint on overall spindle architecture (Crowder et al., 2015). One possible mechanism to explain the differing proportions of MT subpopulations observed in varying cell types could be related to changes in the pathways of MT nucleation.

\section{Mechanisms of spindle microtubule nucleation}

The first and best studied MT organizing center is the centrosome. This organelle is comprised of two centrioles surrounded by an amorphous mass of proteins termed pericentriolar material (PCM) (Urbani and Stearns, 1999). One important component of the PCM is $\gamma$-tubulin, which, together with additional subunits named gamma-tubulin complex proteins (GCPs), form a macromolecular assembly called the $\gamma$-tubulin ring complex $(\gamma$ TuRC). The $\gamma$-TuRC acts as an active MT-nucleator, capping, stabilizing and anchoring the minus-ends to the centrosome (Zheng et al., 1995). With the discovery of MT dynamic instability, Kirschner and Mitchison proposed in 1986 a model for spindle assembly known as "search-and-capture" (Kirschner and Mitchison, 1986). This model suggested that centrosome-nucleated MTs probe space within the cytoplasm until they are captured and stabilized by one of the kinetochores on a chromosome. Thus, chromosomes are firstly captured by only one pole, and are thus "mono-oriented". Then, when the opposite kinetochore captures MTs from the opposite pole, they become "bi-oriented". Search-andcapture was actually directly visualized in live newt lung cells only four years after its 
formulation (Hayden et al., 1990; Rieder and Alexander, 1990). This mechanism relies on dynamic instability, without which the search time would be too long compared to the time it takes a spindle to assemble (Holy and Leibler, 1994). However, because the density of MT ends decreases as the distance between the centrosome and chromosomes increases, searchand-capture of 46 chromosomes in human cells would take much longer than the observed duration of prometaphase, if not biased towards chromosomes (Wollman et al., 2005). This observation implied the existence of mechanisms facilitating the encounter between microtubules and kinetochores to promote spindle assembly.

With the development of cell-free Xenopus egg extracts (Lohka and Masui, 1983), spindle assembly could be observed in an in vitro system through the addition of demembranated Xenopus sperm nuclei (Lohka and Maller, 1985). This system was then used to discover and study MT nucleation pathways and mechanisms of spindle assembly in a vertebrate model. In this system, addition of plasmid DNA-coated beads lacking both centrosomes and kinetochores was sufficient to form bipolar spindles (Heald et al., 1996). This was consistent with the previous observation that injection of viral DNA into a frog egg promoted microtubule assembly (Karsenti et al., 1984). These studies thus revealed the existence of mechanisms allowing for the nucleation of MTs in the vicinity of the chromosomes. By comparing spindles assembled around sperm nuclei containing centrosomes with those assembled around chromatin beads, Heald and colleagues suggested that, while centrosomes are dispensable for spindle assembly, they regulate the organization of spindle MTs by remaining an important site for spindle pole formation (Heald et al., 1997). Assembly of acentrosomal spindle was described as early as 1972 in mouse oocytes (Szollosi et al., 1972). In Drosophila, spindle assembly was also observed in meiotic female cells in which centrosomes are absent (Theurkauf and Hawley, 1992). Using laser microsurgery to destroy centrosomes in CVG-2 cells (green monkey fibroblasts), Khodjakov and colleagues revealed that spindle were able to self-assemble in the absence of centrosome (Khodjakov et al., 2000). It was moreover demonstrated that cell division and development progressed without defect in fly embryos that contained no centrosomes (Basto et al., 2006). Therefore, chromatin-derived MT nucleation is central to spindle assembly, particularly in the absence of centrosomes, and facilitates search-and-capture (Heald and Khodjakov, 2015). Furthermore, acentrosomal spindle assembly highlights the role of MT motors in driving spindle self-organization and bipolarity (Walczak et al., 1998; Gatlin and Bloom, 2010) (Figure 1). 
Several factors are involved in chromosome-dependent MT nucleation and spindle assembly, but one of the key proteins is the small guanosine triphosphatase (GTPase) Ran. While its role in the nuclear import and export machinery is well established (Görlich, 1997), Ran has been demonstrated over the last 20 years to be a central actor in spindle assembly. Ran activity depends on its nucleotide-binding state (GDP or GTP). It is regulated by the GTPase activating protein (GAP) RanGAP1 in the cytoplasm, and by the guanine nucleotide exchange factor (GEF) RCC1, which is bound to chromatin. RanBP1 (a guanine nucleotide dissociation inhibitor) acts as a cofactor for RanGAP1 by increasing its GTP hydrolysis activity. Kalab, Pu and Dasso showed that spindle assembly is disrupted by the addition of RanBP1 to Xenopus egg extract, showing a role of Ran in spindle assembly, independently of its role as a nuclear transporter (Kalab et al., 1999). Concomitantly, other studies using egg extracts led to the proposition that RCC1 generates a high concentration of RanGTP around chromosomes (Carazo-Salas et al., 1999), which acts as a mechanism for chromosomedependent stimulation of MT nucleation and spindle assembly (Wilde and Zheng, 1999). This hypothesis was then confirmed by the direct visualization of a RCC1 derived RanGTP gradient surrounding chromosomes throughout the cell cycle (Kalab et al., 2002). RCC1 binds directly to histones $\mathrm{H} 2 \mathrm{~A}$ and $\mathrm{H} 2 \mathrm{~B}$ of the nucleosomes that form chromatin. This interaction was suggested to stimulate the activity of RCC1, and thus to establish the polarity of the RanGTP gradient (Nemergut et al., 2001). Remarkably, RCC1-coated beads were sufficient to promote bipolar spindle assembly in Xenopus egg extracts without chromatin or kinetochores (Halpin et al., 2011).

The mechanism by which RanGTP promotes MT nucleation at the proximity of chromosomes involves the release of various spindle assembly factors (SAFs) from sequestration by importins $\alpha$ and $\beta$. RanGTP was shown to act as a spindle assembly stimulator by increasing the rescue frequency of MTs, and by altering the balance of motor activities (Wilde et al., 2001). To date, more than twenty SAFs have been shown to be regulated by the RanGTP gradient and are listed elsewhere (Cavazza and Vernos, 2016). One example of RanGTP regulated SAFs is the spindle MT cross-linking kinesin XCTK2, which requires a RanGTP gradient for proper localization and motility (Weaver et al., 2015). Another important SAF regulated by RanGTP is TPX2, which will be discussed later in more detail. TPX2 is inactivated by importin $\alpha$ and is required for RanGTP activity and chromosome-dependent spindle self-assembly (Gruss et al., 2001). TPX2 is also involved in a process called "MT amplification" that is crucial for the generation of the high density of MTs within the spindle (Figure 1, inset 5) and which is controlled by the Ran gradient (Petry 
et al., 2013). By employing a genome-wide RNAi screen in Drosophila S2 cells for spindle morphology (Goshima et al., 2007), Augmin was identified as a protein complex involved in the recruitment of $\gamma$-TuRC to the side of pre-existing MTs and required for centrosomeindependent MT nucleation within the spindle (Goshima et al., 2008). However, due to the high MT density within the spindle, branching MT nucleation could not be observed. It is only when total internal reflection (TIRF) microscopy was used in Xenopus egg extract that MT branching could be observed for the first time. These experiments showed that MTs grow at a low branch angle that conserves the polarity of the MT from which it is branching, forming parallel MT structures (Petry et al., 2013). More recently, it was demonstrated that TPX2 is first deposited on pre-existing MTs, followed by Augmin and $\gamma$-TuRC, which then nucleates branching MTs (Thawani et al., 2019).

In addition to RanGTP, another factor, whose localized activity functions in a gradient to regulate spindle assembly, is Aurora B ( $\mathrm{Xu}$ et al., 2010). This kinase is part of the Chromosome Passenger Complex (CPC), which is required for chromatin-dependent MT stabilization and spindle assembly (Sampath et al., 2004). While RCC1 localizes throughout the chromatin, the $\mathrm{CPC}$ is concentrated at the inner centromere, and is thus responsible for the generation of most of the MTs near the kinetochores. This complex is also composed of INCENP, survivin, and Borealin/DasraA (Kelly et al., 2007). Aurora B locally phosphorylates and inactivates MT-destabilizing proteins including the kinesin-13/MCAK (Andrews et al., 2004; Lan et al., 2004; Ohi et al., 2004) and the heat-stable oncoprotein Stathmin/Op18 (Gadea and Ruderman, 2006). The importance of the CPC for spindle assembly in the absence of centrosomes was first demonstrated in Drosophila female meiosis (Colombié et al., 2008). Using Xenopus egg extracts in the absence of RanGTP-gradient, the activity of the CPC was shown to be sufficient to assemble bipolar spindles (Maresca et al., 2009). These two gradients are thus essential for bipolar spindle formation and originate from mitotic chromatin in the case of Ran, or from centromeric chromatin in the case of CPC. While these pathways seem to be distinct (Sampath et al., 2004; Maresca et al., 2009), relationships exist between the two. For instance, $\mathrm{RCC} 1$ acts at the kinetochore to modulate the spindle assembly checkpoint (Arnaoutov and Dasso, 2003), where it regulates the metaphase-anaphase transition through the displacement of Sgol and CPC (Zhang et al., 2018). In addition, the stabilization of k-fiber minus-ends is ensured by a Ran-GTPdependent mechanism (Meunier and Vernos, 2011) and Ran-GTP is crucial for the maintenance of stable kinetochore-microtubule attachments through the regulation of Aurora 
B (Lee et al., 2012). It is also interesting to note that RanBP2 is a regulator of Borealin sumoylation, a modification peaking in early mitosis (Klein et al., 2009).

\section{Physical constraints influencing spindle size}

While there have been numerous studies regarding mechanisms of spindle assembly, it is only recently that the range of spindle sizes and morphologies and their regulation have begun to be appreciated. By performing a comparative analysis of spindle morphometrics across metazoans, Crowder et al. revealed a linear scaling of spindle size to embryonic cell size conserved across phyla (Crowder et al., 2015). Interestingly, they also revealed a moderate contribution of genome size to spindle size. One interesting question is whether the scaling of spindle size to cell and genome size is the consequence of direct physical constraints, or is due to evolutionary driven molecular adaptations.

In the 1980's, Nicklas et al. demonstrated for the first time a direct relationship between chromosome number and spindle length (Nicklas and Gordon, 1985). Using micromanipulation and electron microscopy on spermatocytes from Melanoplus differentialis, they showed that the number of chromosomes determines spindle length, as the one by one removal of chromosomes resulted in a gradual decrease in spindle length, until it reached $\sim 40 \%$ of its original size after all chromosomes were removed. Consistent with a scaling relationship between genome and spindle size, a strong correlation between organism, cell, spindle, and genome size exists in two Xenopus specie. X. laevis possesses an allotetraploid genome and a large spindle, while $X$. tropicalis has a diploid genome and a smaller spindle. Using chromatin-coated beads with a controlled quantity of DNA per bead and Xenopus egg extract to assemble spindles, it was shown that the chromatin mass influences in a direct manner the size of the spindle, but only minimally with a size increase of only $10 \%$ despite a $400 \%$ increase in the amount of DNA (Dinarina et al., 2009). Consistent with this observation, it was found that $X$. laevis spindles assembled around $X$. tropicalis chromosomes (half the genome size) were only $10 \%$ shorter in length (Brown et al., 2007). Similarly, by generating haploid $X$. laevis embryos, it was shown that mitotic spindle size can be reduced by about only $10 \%$ by halving the amount of DNA in embryos (Wühr et al., 2008). Finally, in C. elegans, spindle morphology adjusts to ploidy, with the exception that an increase in ploidy is

accommodated by an increase in spindle width rather than length (Hara and Kimura, 2013). Therefore, while genome size seems to directly influence spindle size, its contribution is quite 
limited, is not linear, and does not explain the total spindle length differences between species.

Another physical factor that might contribute to spindle scaling is cell size. Using microfluidic technology to encapsulate cytoplasm in defined volumes and geometries, it was demonstrated that varying the cytoplasmic volume is sufficient to change spindle length, and to mimic the scaling observed in Xenopus cells during development (Good et al., 2013; Hazel et al., 2013). Notably, these experiments also reproduced the upper size limit observed earlier in Xenopus (Wühr et al., 2008) and in other metazoans (Crowder et al., 2015), so that the spindle starts to scale only when compartment size is reduced to $\sim 140 \mathrm{~mm}$ in diameter. These studies confirmed the existence of two regimes of spindle scaling to cell size: one in which spindle size is physically coupled to cell diameter, and a second in which they are uncoupled. To explain the coupling of spindle size to cell size, Good et al. proposed the limiting component model: by reducing cytoplasmic volume, one component becomes quantitatively limiting to form the spindle, leading to a decrease in spindle size (Good et al., 2013). A previous report suggested that the amount of available tubulin determines spindle length (Sluder, 1976). Good et al. tested tubulin as a possible limiting factor but supplementing the extract with brain tubulin did not alter spindle scaling in droplets, which led to the suggestion that the levels of several factors could be limiting for spindle assembly (Good et al., 2013). Interestingly, experimental addition of the MT polymerase XMAP215 (factor discussed below) to Xenopus egg extracts and embryos was enough to increase spindle size increase (Reber et al., 2013; Milunovic-Jevtic et al., 2018). This suggests that while other spindle components, such as tubulin, are not limiting, XMAP215 might be. This is actually interesting to note that MT growth velocity was shown to correlate with embryonic mitotic spindle length in C. elegans and sea urchin. In these systems, MT growth speed decreased with decreasing cell size, supporting a model in which spindle size could be set by a limiting MT polymerase (Lacroix et al., 2018). In apparent contradiction with the idea of tubulin not being limiting, it was recently shown that addition of Xenopus tubulin, but not brain tubulin, is able to increase spindle size and mass in egg extract (Hirst et al., 2020).

While physical constraints appear to contribute to spindle scaling, it has become clear that these mechanisms are superimposed upon molecular controls. Indeed, different spindle scaling was observed in droplets of the same size with different stage $X$. laevis embryo extracts (early vs later stages), revealing that cytoplasmic composition also contributes to spindle scaling (Good et al., 2013). Helping to explain this phenomenon, importin $\alpha$ has recently been shown to be a sensor of the surface area and volume of the cell in order to 
regulate organelle size scaling, including the spindle, by modulating the activity of regulator proteins through the interaction with their nuclear localization sequences (Brownlee and Heald, 2019).

\section{Molecular control of spindle length through modulation of MT dynamics, transport and nucleation}

Interest in spindle length regulation started gaining traction through multiple studies in the early 2000s. With the first systematic evaluation of MT dynamics factors on metaphase spindle length control performed in a Drosophila S2 cell line, Goshima and colleagues showed that spindle length was sensitive to alterations in MT dynamics with a decrease in length with the depletion of MT stabilizers (EB1, Msps/chTOG, Mast/CLASP) or an increase in length with the depletion of depolymerizers (Kinesin-13 Klp10A, Kinesin-8 Klp67A) as well as opposite effects through the overexpression of these factors (Goshima et al., 2005) (Figure 2A). Using computer simulations, more recent studies clearly exemplified that modulating MT dynamics is sufficient to control spindle length and to recapitulate observed variations (Loughlin et al., 2010; Lacroix et al., 2018; Hirst et al., 2020). One interesting example of MT regulators controlling spindle size is XMAP215/chTOG. XMAP215 was first described as a Xenopus MT-Associated Protein (XMAP) that promotes an increase in the MT growth rate in vitro (Gard and Kirschner, 1987), and then, more precisely, as a processive polymerase able to directly add up to 25 tubulin dimers at a time to the MT plus-end (Brouhard et al., 2008). Moreover, it was shown in Xenopus egg extracts to control spindle length, with increasing MT growth velocity being linearly correlated with an increase in spindle length and total MT spindle mass (Reber et al., 2013). However, because MT density, lifetime and spindle shape remained unchanged, the authors proposed the idea that spindle size is determined by a mass balance, whereas shape is determined by a force balance (Reber et al., 2013). Consistent with this work in egg extract, microinjection of XMAP215 in $X$. laevis embryo cells demonstrated that it is sufficient to induce larger spindles in cells, confirming its role in spindle length control (Milunovic-Jevtic et al., 2018). Yet, this MTpolymerizer does not seem to act alone. Indeed, its interaction with EB1 (end-binding protein 1) appears to be necessary for spindle organization as its disruption results in shorter and multipolar spindles, with reduced MT density, as well as failure in chromosome segregation (Kronja et al., 2009). Other factors that stabilize MTs were shown to participate to spindle length control. This is the case of the chromokinesin Kid that bundles and stabilizes MTs 
(Tokai-Nishizumi et al., 2005; Bisht et al., 2019) and of other factors of the TOG-like domain containing-protein family CLASP/Mast/Orbit that act at the MT plus-end to stabilize them (Maiato et al., 2005; Hannak and Heald, 2006; Laycock et al., 2006; Reis et al., 2009; Espiritu et al., 2012; Young et al., 2014), both of these families being in fact primarily important for spindle integrity. In addition, MT depolymerases or destabilizers, such as the Kinesin-13 MCAK (Mitchison et al., 2005), the MT destabilizing protein Stathmin/Op18 (Budde et al., 2001), and of various MT depolymerases of the Kinesin-8 family (Savoian and Glover, 2010; Wang et al., 2010; Stout et al., 2011; Weaver et al., 2011) were also shown to be involved in spindle length control in particular in Xenopus and invertebrate systems.

Another important mechanism through which spindle size can be adjusted is MT translocation and sliding by molecular motors (Figure 2B). Several studies in yeast and drosophila revealed the role of antagonistic MT sliding mechanisms of in particular Kinesins -5 and -14 in controlling spindle length (Saunders et al., 1997; Goshima et al., 2005; BrustMascher et al., 2009; Goshima and Scholey, 2010; Syrovatkina et al., 2013). In Xenopus, the minus end-directed Kinesin-14 motor XCTK2 was shown to control metaphase spindle length (Cai et al., 2009). This motor cross-links and slides both parallel and antiparallel MTs (Tao et al., 2006; Hentrich and Surrey, 2010) and contributes to proper spindle assembly (Walczak et al., 1997, 1998). XCTK2 MT binding is regulated by the Ran-GTP gradient through the association of importin $\alpha / \beta$ with a bipartite nuclear localization sequence located in the tail of XCTK2 (Ems-mcclung et al., 2004). This association allows the Ran-GTP gradient to spatially coordinate the motility and the localization of XCTK2 within the spindle (Weaver et al., 2015). In addition, while XTCK2 is able to cross-link both parallel and antiparallel MTs, the Ran-GTP gradient generates an effector gradient of XCTK2 that leads to the preferential parallel MT cross-linking and sliding near the spindle poles, thus participating to their focusing (Ems-McClung et al., 2020). Interestingly, the cross-linking and sliding activities of HSET/XTCK2 are required to regulate spindle length and the RanGTP regulation of these activities is important for proper spindle morphology (Cai et al., 2009). This led the authors to propose that the Kinesin-14 motors would cross-link and transport parallel MTs in a half spindle, not only to facilitate pole focusing, but also for spindle elongation.

Finally, recent studies suggest that MT nucleation may also regulate spindle length with a key role of the TPX2 protein (Figure 2C). In C. elegans, centrosome size was shown to scale with spindle size. While this was independent of $\gamma$-tubulin levels, centrosome size determined the length of a TPXL-1 (TPX2-Like-1) gradient along MTs, resulting in the 
control of spindle length (Greenan et al., 2010). TPX2 was discovered as being responsible for the localization of Xklp2, a plus-end-directed kinesin-like needed for centrosome separation at the onset of spindle formation (Wittmann et al., 1998). The interest in TPX2 (targeting protein for Xklp2) increased with the discovery of its role in the organization of spindle poles (Wittmann et al., 2000). Recent findings highlighted TPX2 as a determinant of spindle shape in murine neural stem cells, contributing to robustness and correct chromosome segregation (Vargas-Hurtado et al., 2019). In HeLa cells, TPX2 is essential for the formation of a stable bipolar chromosome-induced spindle (Gruss et al., 2002), and its reduced expression by siRNA leads to multipolar spindles and fragmented centrosomes (Garrett et al., 2002). Notably, TPX2 has been shown in this system to bind to CLASP and the kinesin-13 Kif2a, what appears to control spindle length (Fu et al., 2015). Importantly, TPX2 functions in spindle size control through a central role in MT nucleation from pre-existing MTs via the augmin pathway and this nucleation potential is increased by Ran-GTP (Petry et al., 2013; Alfaro-Aco et al., 2017; Thawani et al., 2019). It was recently shown that in Xenopus egg extract the upper spindle size limit is determined by the spatial regulation of this MTstimulated MT nucleation (Decker et al., 2018). Yet, while TPX2 is regulated by Ran-GTP, it was shown that the interaction between SAFs and microtubules leads to a feedback mechanism that amplifies spindle assembly factors to make spindle size independent of the RanGTP gradient (Oh et al., 2016).

\section{Molecular mechanisms of spindle size scaling in Xenopus}

MT dynamics, transport and nucleation are all accepted to be potentially involved in spindle scaling. Xenopus frogs have been instrumental over the past 10 years in investigating mechanisms of interspecies and developmental scaling. These studies revealed mechanisms of vertebrate spindle scaling through the regulation of MT dynamics by the severing factor Katanin (Loughlin et al., 2010, 2011) and the depolymerizing kinesin Kif2a (Wilbur and Heald, 2013; Brownlee and Heald, 2019; Miller et al., 2019) as well as regulation of MT nucleation and translocation by a TPX2/Eg5 module (Helmke and Heald, 2014) (Figure 3).

Katanin was first described as an enzyme that uses the energy of ATP hydrolysis to sever MTs by breaking tubulin-tubulin bonds (McNally and Vale, 1993). Subsequent studies have revealed its role to be more complex, and Katanin has proposed to regulate the density of MTs by increasing their number and decreasing their length, as well as by increasing the number of non-centrosomal MTs through its severing activity (Buster et al., 2002). In $C$. elegans meiotic cells, katanin was shown to convert long MTs into shorter ones near meiotic 
chromosomes, while a decrease in katanin in mutant cells inhibited the self-organization of MTs into a bipolar spindle (Srayko et al., 2006). A role for katanin in interspecies spindle scaling was revealed by Loughlin et al., who found that an inhibitory Aurora-B phosphorylation site in the catalytic subunit contributes to the spindle size difference observed between two Xenopus species. Indeed, this site is present in X. laevis, while absent from $X$. tropicalis. By combining in silico simulations with inhibition of katanin in egg extracts, they concluded that increased katanin activity in $X$. tropicalis results in a shorter spindle than in X. laevis (Loughlin et al., 2010, 2011).

Kif2a is a kinesin-13 MT-depolymerizing protein that interacts with MTs. Its activity results in shorter MTs, and is inhibited by importin $\alpha$ binding (Wilbur and Heald, 2013). During development, with decreasing cell size directly leading to an increased surface area to cell volume ratio, importin $\alpha$ membrane sequestration increases, resulting in the release of Kif2a inhibition and thus smaller spindles. This process was shown to regulate the developmental scaling of $X$. laevis spindles and nuclei as well as spindle and nuclear scaling in somatic human cells (Brownlee and Heald, 2019). Interestingly, Kif2a was also shown to participate in spindle scaling in a distantly related Pipid frog, Hymenochirus boettgeri. In this species, Kif2a possesses a Plk1 phosphorylation site, not present in X. laevis, which allows its activation (Miller et al., 2019), thus participating in interspecies scaling. Moreover, kif2a has also been shown to be involved in spindle self-organization by being spatially-regulated by the Aurora B gradient (Uehara et al., 2013), and to be targeted to spindle poles by dynein/dynactin to promote local MT depolymerization and spindle length regulation (Gaetz and Kapoor, 2004).

Remarkably, in addition to such molecular controls of MT dynamics that regulate spindle size, a recent study revealed that differences in intrinsic dynamic properties of tubulin itself contribute to spindle length difference between X. laevis and X. tropicalis (Hirst et al., 2020).

In addition to its role in MT nucleation, the TPX2 protein interacts with Aurora A by its N-terminal domain (Brunet et al., 2004) and with the kinesin-5 Eg5 with its C-terminal domain (Eckerdt et al., 2008). Bacterial Artificial Chromosome (BAC) technology (Kittler et $a l ., 2005)$ has been used to investigate the interaction between these factors by generating TPX2 lacking its Eg5 interacting domain. This work showed that this interaction is involved in k-fiber formation and spindle organization, but more importantly, that TPX2 localizes Eg5 to spindle poles and regulates its activity in MT sliding (Ma et al., 2011). In fact, Eg5 was first described in Aspergillus nidulans as a kinesin-like involved in nuclear division (Enos 
and Morris, 1990). While its role in MT sliding at the spindle midzone is well known (Kapitein et al., 2005), its involvement in spindle architecture is still being investigated. Spindle flux, which corresponds to the continuous poleward movement of tubulin, relies on the constant addition of tubulin subunits at microtubule plus ends and their corresponding removal from microtubule minus-ends at spindle poles as well as MT sliding towards the poles. This latter aspect is thought to be regulated by Eg5 since its inhibition or its depletion result in decreasing flux rates (Miyamoto et al., 2004). Interestingly, careful analysis of the flux velocity revealed that it decreases by about $20 \%$ near the poles and that the inhibition of either or both Eg5 and dynein/dynactin leads to the suppression of this slow-down (Yang et $a l ., 2008)$. It was recently proposed that a specific array of MTs in the mid half-spindle possesses less mechanical rigidity than elsewhere, behaving in a fluid-like manner, and that this heterogeneity relies upon Eg5 and dynein (Takagi et al., 2019). Importantly, a TPX2/Eg5 interaction was shown to be involved in controlling spindle length and architecture and responsible for the differences observed between $X$. laevis and $X$. tropicalis spindles. Indeed, Helmke and Heald found that TPX2 levels were three times higher in X. tropicalis cytoplasm compared to $X$. laevis. Sufficiently, addition of TPX2 in X. laevis extracts reduced spindle length by about $20 \%$ and relocalized Eg5 to the spindle poles similar to X. tropicalis spindles. Interestingly, TPX2 contains a stretch of 7 amino acids only present in X. laevis, and while its deletion in X. laevis increases MT nucleation, its insertion in X. tropicalis decreases MT nucleation (Helmke and Heald, 2014). Therefore, TPX2 appears to be a key factor which modulates MT nucleation, organization, and spindle assembly, the total sum of which is used to scale spindle size.

\section{Conclusion}

One hundred and forty years after the first description of mitosis, we are finally beginning to obtain a clearer picture of the organization of the spindle and are even approaching a mechanistic understanding of the establishment of spindle architecture. The study of the spindle at the molecular level benefited greatly from the development of new molecular and imaging technologies during the past few decades. In particular, Xenopus extract systems have increasingly been instrumental in discovering mechanisms of spindle assembly, while recent interspecies studies have allowed for a deeper understanding of spindle architecture variations. Yet, it remains to be assessed what molecular players are universally at work or specific to a particular species or cell. Moreover, in addition to responding to numerous molecular factors, it becomes clear that spindle assembly also responds to genome and cell 
size to scale and optimize its function to a particular cellular environment. Overall, as we decipher mechanisms by which spindles assemble and organize, understanding the interplay between the different mechanisms and pathways, and how they contribute to faithful chromosome segregation will likely require many more years of investigation.

\section{Acknowledgements}

We thank the members of "Tubulin and Interacting Proteins" team for their support and, in particular, Dr. Denis Chrétien and Dr. Laurence Duchesne for suggestions on the manuscript. We are also grateful to Prof. Christopher W. Brownlee (Stony Brook University School of Medicine), Prof. Rebecca Heald (University of California, Berkeley) as well as the two reviewers for critical reading and helpful suggestions to the manuscript. We finally apologize to all those whose work could not be cited due to space limitations.

\section{Funding}

RG was supported by an HFSP Career Development Award CDA00019/2019-C and GG by a Master fellowship from the French Society for Microscopies. 


\section{References}

Alfaro-Aco, R, Thawani, A, and Petry, S (2017). Structural analysis of the role of TPX2 in branching microtubule nucleation. J Cell Biol 216, 983-997.

Andrews, PD, Ovechkina, Y, Morrice, N, Wagenbach, M, Duncan, K, Wordeman, L, and Swedlow, JR (2004). Aurora B regulates MCAK at the mitotic centromere. Dev Cell 6, 253268.

Arnaoutov, A, and Dasso, M (2003). The Ran GTPase regulates kinetochore function. Dev Cell 5, 99-111.

Barisic, M, Silva e Sousa, R, Tripathy, SK, Magiera, MM, Zaytsev, A V., Pereira, AL, Janke, C, Grishchuk, EL, and Maiato, H (2015). Microtubule detyrosination guides chromosomes during mitosis. Science (80- ) 348, 799-803.

Basto, R, Lau, J, Vinogradova, T, Gardiol, A, Woods, CG, Khodjakov, A, and Raff, JW (2006). Flies without Centrioles. Cell 125, 1375-1386.

Bennabi, I, Terret, ME, and Verlhac, MH (2016). Meiotic spindle assembly and chromosome segregation in oocytes. J Cell Biol 215, 611-619.

Bisht, JS, Tomschik, M, and Gatlin, JC (2019). Induction of a Spindle-Assembly-Competent M Phase in Xenopus Egg Extracts. Curr Biol 29, 1273-1285.e5.

Brinkley, BR, and Cartwright, J (1971). Ultrastructural analysis of mitotic spindle elongation in mammalian cells in vitro. Direct microtubule counts. J Cell Biol 50, 416-431.

Brouhard, GJ, Stear, JH, Noetzel, TL, Al-Bassam, J, Kinoshita, K, Harrison, SC, Howard, J, and Hyman, AA (2008). XMAP215 is a processive microtubule polymerase. Cell 132, 79-88. Brown, KS, Blower, MD, Maresca, TJ, Grammer, TC, Harland, RM, and Heald, R (2007). Xenopus tropicalis egg extracts provide insight into scaling of the mitotic spindle. J Cell Biol $176,765-770$.

Brownlee, C, and Heald, R (2019). Importin a Partitioning to the Plasma Membrane Regulates Intracellular Scaling. Cell 176, 805-815.e8.

Brunet, S, Sardon, T, Zimmerman, T, Wittmann, T, Pepperkok, R, Karsenti, E, and Vernos, I (2004). Characterization of the TPX2 Domains Involved in Microtubule Nucleation and Spindle Assembly in Xenopus Egg Extracts. Mol Biol Cell 15, 5318-5328.

Brust-Mascher, I, Sommi, P, Cheerambathur, DK, and Scholey, JM (2009). Kinesin-5dependent Poleward Flux and Spindle Length Control in Drosophila Embryo Mitosis. Mol Biol Cell 20, 1749-1762.

Budde, PP, Kumagai, A, Dunphy, WG, and Heald, R (2001). Regulation of Op18 during 
spindle assembly in Xenopus egg extracts. J Cell Biol 153, 149-157.

Buster, D, McNally, K, and McNally, FJ (2002). Katanin inhibition prevents the redistribution of gamma-tubulin at mitosis. J Cell Sci 115, 1083-1092.

Cai, S, Weaver, LN, Ems-McClung, SC, and Walczak, C (2009). Kinesin-14 Family Proteins HSET/XCTK2 Control Spindle Length by Cross-Linking and Sliding Microtubules. Mol Biol Cell 20, 1348-1359.

Carazo-Salas, RE, Guarguaglini, G, Gruss, OJ, Segref, A, Karsenti, E, and Mattaj, IW (1999). Generation of GTP-bound Ran by RCC1 is required for chromatin-induced mitotic spindle formation. Nature 400, 178-181.

Cavazza, T, and Vernos, I (2016). The RanGTP Pathway: From Nucleo-Cytoplasmic Transport to Spindle Assembly and Beyond. Front Cell Dev Biol 3.

Chang, P, Jacobson, MK, and Mitchison, TJ (2004). Poly(ADP-ribose) is required for spindle assembly and structure. Nature 432, 645-649.

Cheeseman, IM, and Desai, A (2008). Molecular architecture of the kinetochore-microtubule interface. Nat Rev Mol Cell Biol 9, 33-46.

Chunduri, NK, and Storchová, Z (2019). The diverse consequences of aneuploidy. Nat Cell Biol 21, 54-62.

Colombié, N, Cullen, CF, Brittle, AL, Jang, JK, Earnshaw, WC, Carmena, M, McKim, K, Ohkura, H, and Colombie, $N$ (2008). Dual roles of incenp crucial to the assembly of the acentrosomal metaphase spindle in female meiosis. Development 135, 3239-3246.

Crowder, ME, Strzelecka, M, Wilbur, JD, Good, MC, Von Dassow, G, and Heald, R (2015). A comparative analysis of spindle morphometrics across metazoans. Curr Biol 25, 15421550 .

Decker, F, Oriola, D, Dalton, B, and Brugués, J (2018). Autocatalytic microtubule nucleation determines the size and mass of Xenopus laevis egg extract spindles. Elife 7, 1-20.

Dinarina, A, Pugieux, C, Corral, MM, Loose, M, Spatz, J, Karsenti, E, and Nédélec, F (2009). Chromatin Shapes the Mitotic Spindle. Cell 138, 502-513.

Eckerdt, F, Eyers, PA, Lewellyn, AL, Prigent, C, and Maller, JL (2008). Spindle pole regulation by a discrete Eg5-interacting domain in TPX2. Curr Biol 18, 519-525.

Ems-McClung, SC, Emch, M, Zhang, S, Mahnoor, S, Weaver, LN, and Walczak, C (2020). RanGTP induces an effector gradient of XCTK2 and importin $\alpha / \beta$ for spindle microtubule cross-linking. J Cell Biol 219, 1-15.

Ems-mcclung, SC, Zheng, Y, and Walczak, C (2004). Importin alpha/bêta and Ran-GTP Regulate XCTK2 Microtubule Binding through a Bipartite Nuclear Localization Signal. Mol 
Biol Cell 15, 46-57.

Enos, A, and Morris, NR (1990). Mutation of a gene that encodes a kinesin-like protein blocks nuclear division in A. nidulans. Cell 60, 1019-1027.

Espiritu, EB, Krueger, LE, Ye, A, and Rose, LS (2012). CLASPs function redundantly to regulate astral microtubules in the C. elegans embryo. Dev Biol 368, 242-254.

Flemming, W (1882). Zellsubstanz, Kern und Zelltheilung. Leipzig, F C W Vogel.

Fu, J, Bian, M, Xin, G, Deng, Z, Luo, J, Guo, X, Chen, H, Wang, Y, Jiang, Q, and Zhang, C (2015). TPX2 phosphorylation maintains metaphase spindle length by regulating microtubule flux. J Cell Biol 210, 373-383.

Gadea, BB, and Ruderman, J V. (2006). Aurora B is required for mitotic chromatin-induced phosphorylation of Op18/Stathmin. Proc Natl Acad Sci U S A 103, 4493-4498.

Gaetz, J, and Kapoor, TM (2004). Dynein/dynactin regulate metaphase spindle length by targeting depolymerizing activities to spindle poles. J Cell Biol 166, 465-471.

Gard, DL, and Kirschner, MW (1987). A microtubule-associated protein from Xenopus eggs that specifically promotes assembly at the plus-end. J Cell Biol 105, 2203-2215.

Garrett, S, Auer, K, Compton, DA, and Kapoor, TM (2002). hTPX2 Is Required for Normal Spindle Morphology and Centrosome Integrity during Vertebrate Cell Division. Curr Biol 12, 2055-2059.

Gatlin, JC, and Bloom, K (2010). Microtubule motors in eukaryotic spindle assembly and maintenance. Semin Cell Dev Biol 21, 248-254.

Good, MC, Vahey, MD, Skandarajah, A, Fletcher, D a., and Heald, R (2013). Cytoplasmic volume modulates spindle size during embryogenesis. Science 342, 856-860.

Görlich, D (1997). Nuclear protein import. Curr Opin Cell Biol 9, 412-419.

Goshima, G, Mayer, M, Zhang, N, Stuurman, N, and Vale, RD (2008). Augmin: a protein complex required for centrosome-independent microtubule generation within the spindle. $\mathrm{J}$ Cell Biol 181, 421-429.

Goshima, G, and Scholey, JM (2010). Control of Mitotic Spindle Length. Annu Rev Cell Dev Biol 26, 21-57.

Goshima, G, Wollman, R, Goodwin, SS, Zhang, N, Scholey, JM, Vale, RD, and Stuurman, N (2007). Genes Required for Mitotic Spindle Assembly in Drosophila S2 Cells. Science (80- ) $316,417-421$.

Goshima, G, Wollman, R, Stuurman, N, Scholey, JM, and Vale, RD (2005). Length Control of the Metaphase Spindle. Curr Biol 15, 1979-1988.

Greenan, G, Brangwynne, CP, Jaensch, S, Gharakhani, J, Jülicher, F, and Hyman, A (2010). 
Centrosome size sets mitotic spindle length in Caenorhabditis elegans embryos. Curr Biol 20, $353-358$.

Groen, AC, Coughlin, M, and Mitchison, TJ (2011). Microtubule assembly in meiotic extract requires glycogen. Mol Biol Cell 22, 3139-3151.

Gruss, OJ, Carazo-Salas, RE, Schatz, CA, Guarguaglini, G, Kast, J, Wilm, M, Le Bot, N, Vernos, I, Karsenti, E, and Mattaj, IW (2001). Ran induces spindle assembly by reversing the inhibitory effect of importin alpha on TPX2 activity. Cell 104, 83-93.

Gruss, OJ, Wittmann, M, Yokoyama, H, Pepperkok, R, Kufer, T, Silljé, H, karsenti, E, Mattaj, IW, and Vernos, I (2002). Chromosome-induced microtubule assembly mediated by TPX2 is required for spindle formation in HeLa cells. Nat Cell Biol 4, 871-879.

Halpin, D, Kalab, P, Wang, J, Weis, K, and Heald, R (2011). Mitotic spindle assembly around RCC1-coated beads in Xenopus egg extracts. PLoS Biol 9, e1001225.

Hannak, E, and Heald, R (2006). Xorbit/CLASP links dynamic microtubules to chromosomes in the Xenopus meiotic spindle. J Cell Biol 172, 19-25.

Hara, Y, and Kimura, A (2013). An allometric relationship between mitotic spindle width, spindle length, and ploidy in Caenorhabditis elegans embryos. Mol Biol Cell 24, 1411-1419. Hayden, JH, Bowser, SS, and Rieder, CL (1990). Kinetochores capture astral microtubules during chromosome attachment to the mitotic spindle: Direct visualization in live newt lung cells. J Cell Biol 111, 1039-1045.

Hazel, J, Krutkramelis, K, Mooney, P, Tomschik, M, Gerow, K, Oakey, J, and Gatlin, JC (2013). Changes in cytoplasmic volume are sufficient to drive spindle scaling. Science 342, $853-856$.

Heald, R, and Khodjakov, A (2015). Thirty years of search and capture: The complex simplicity of mitotic spindle assembly. J Cell Biol 211, 1103-1111.

Heald, R, Tournebize, R, Blank, T, Sandaltzopoulos, R, Becker, P, Hyman, A, and Karsenti, E (1996). Self-organization of microtubules into bipolar spindles around artificial chromosomes in Xenopus egg extracts. Nature 382, 420-425.

Heald, R, Tournebize, R, Habermann, A, Karsenti, E, and Hyman, A (1997). Spindle assembly in Xenopus egg extracts: respective roles of centrosomes and microtubule selforganization. J Cell Biol 138, 615-628.

Helmke, KJ, and Heald, R (2014). TPX2 levels modulate meiotic spindle size and architecture in Xenopus egg extracts. J Cell Biol 206, 385-393.

Hentrich, C, and Surrey, T (2010). Microtubule organization by the antagonistic mitotic motors kinesin-5 and kinesin-14. J Cell Biol 189, 465-480. 
Hirst, WG, Biswas, A, Mahalingan, KK, and Reber, S (2020). Differences in Intrinsic Tubulin Dynamic Properties Contribute to Spindle Length Control in Xenopus Species. Curr Biol 30, 1-7.

Holy, TE, and Leibler, S (1994). Dynamic instability of microtubules as an efficient way to search in space. Proc Natl Acad Sci 91, 5682-5685.

Inoué, S, and Sato, H (2008). Cell motility by labile association of molecules: The nature of mitotic spindle fibers and their role in chromosome movement. Collect Work Shinya Inoue Microsc Living Cells, Dyn Mol 50, 263-298.

Janke, C, and Bulinski, JC (2011). Post-translational regulation of the microtubule cytoskeleton: mechanisms and functions. Nat Rev Mol Cell Biol 12, 773-786.

Kajtez, J et al. (2016). Overlap microtubules link sister k-fibres and balance the forces on bioriented kinetochores. Nat Commun 7, 10298.

Kalab, P, Pu, RT, and Dasso, M (1999). The ran GTPase regulates mitotic spindle assembly. Curr Biol 9, 481-484.

Kalab, P, Weis, K, and Heald, R (2002). Visualization of a Ran-GTP gradient in interphase and mitotic Xenopus egg extracts. Science 295, 2452-2456.

Kapitein, LC, Peterman, EJG, Kwok, BH, Kim, JH, Kapoor, TM, and Schmidt, CF (2005). The bipolar mitotic kinesin Eg5 moves on both microtubules that it crosslinks. Nature 435, $114-118$.

Karsenti, E, Newport, J, and Kirschner, M (1984). Respective roles of centrosomes and chromatin in the conversion of microtubule arrays from interphase to metaphase. J Cell Biol $99,47 \mathrm{~s}-54 \mathrm{~s}$.

Kelly, AE, Sampath, SC, Maniar, TA, Woo, EM, Chait, BT, and Funabiki, H (2007). Chromosomal Enrichment and Activation of the Aurora B Pathway Are Coupled to Spatially Regulate Spindle Assembly. Dev Cell 12, 31-43.

Kirschner, M, and Mitchison, T (1986). Beyond self-assembly: From microtubules to morphogenesis. Cell 45, 329-342.

Kittler, R, Pelletier, L, Ma, C, Poser, I, Fischer, S, Hyman, AA, and Buchholz, F (2005). RNA interference rescue by bacterial artificial chromosome transgenesis in mammalian tissue culture cells. Proc Natl Acad Sci 102, 2396-2401.

Klein, UR, Haindl, M, Nigg, EA, and Muller, S (2009). RanBP2 and SENP3 Function in a Mitotic SUMO2/3 Conjugation-Deconjugation Cycle on Borealin. Mol Biol Cell 20, 410418.

Kronja, I, Kruljac-Letunic, A, Caudron-Herger, M, Bieling, P, and Karsenti, E (2009). 
XMAP215-EB1 Interaction Is Required for Proper Spindle Assembly and Chromosome Segregation in Xenopus Egg Extract. Mol Biol Cell 20, 2684-2696.

Lacroix, B et al. (2018). Microtubule Dynamics Scale with Cell Size to Set Spindle Length and Assembly Timing. Dev Cell 45, 496-511.e6.

Lan, W, Zhang, X, Kline-Smith, SL, Rosasco, SE, Barrett-Wilt, GA, Shabanowitz, J, Hunt, DF, Walczak, C, and Stukenberg, PT (2004). Aurora B phosphorylates centromeric MCAK and regulates its localization and microtubule depolymerization activity. Curr Biol 14, $273-$ 286.

Laycock, JE, Savoian, MS, and Glover, DM (2006). Antagonistic activities of Klp10A and orbit regulate spindle length, bipolarity and function in vivo. J Cell Sci 119, 2354-2361.

Lee, YP, Wong, CH, Chan, KS, Lai, SK, Koh, CG, and Li, HY (2012). In Vivo FRET Imaging Revealed a Regulatory Role of RanGTP in Kinetochore-Microtubule Attachments via Aurora B Kinase. PLoS One 7, 1-13.

Lohka, MJ, and Masui, Y (1983). Formation in vitro of sperm pronuclei and mitotic chromosomes induced by amphibian ooplasmic components. Science 220, 719-721.

Lohka, MJI, and Maller, JL (1985). Induction of nuclear envelope breakdown, chromosome condensation, and spindle formation in cell-free extracts. J Cell Biol 101, 518-523.

Loughlin, R, Heald, R, and Nédélec, F (2010). A computational model predicts Xenopus meiotic spindle organization. J Cell Biol 191, 1239-1249.

Loughlin, R, Wilbur, JD, McNally, FJ, Nédélec, FJFJ, and Heald, R (2011). Katanin contributes to interspecies spindle length scaling in Xenopus. Cell 147, 1397-1407.

Ma, N, Titus, J, Gable, A, Ross, JL, and Wadsworth, P (2011). TPX2 regulates the localization and activity of Eg5 in the mammalian mitotic spindle. J Cell Biol 195, 87-98.

Maiato, H, Khodjakov, A, and Rieder, CL (2005). Drosophila CLASP is required for the incorporation of microtubule subunits into fluxing kinetochore fibres. Nat Cell Biol 7, 42-47. Maresca, TJ, Groen, AC, Gatlin, JC, Ohi, R, Mitchison, TJ, and Salmon, ED (2009). Spindle assembly in the absence of a RanGTP gradient requires localized CPC activity. Curr Biol 19, $1210-1215$.

Masuda, H, Hirano, T, Yanagida, M, and Cande, WZ (1990). In vitro reactivation of spindle elongation in fission yeast nuc2 mutant cells. J Cell Biol 110, 417-425.

McNally, FJ, and Vale, RD (1993). Identification of katanin, an ATPase that severs and disassembles stable microtubules. Cell 75, 419-429.

Meunier, S, and Vernos, I (2011). K-fibre minus ends are stabilized by a RanGTP-dependent mechanism essential for functional spindle assembly. Nat Cell Biol 13, 1406-1414. 
Miller, KE, Session, AM, and Heald, R (2019). Kif2a Scales Meiotic Spindle Size in Hymenochirus boettgeri. Curr Biol 29, 3720-3727.e5.

Milunovic-Jevtic, A et al. (2018). In vivo mitotic spindle scaling can be modulated by changing the levels of a single protein: the microtubule polymerase XMAP215. Mol Biol Cell $29,1311-1317$.

Mitchison, T, and Kirschner, M (1984). Dynamic instability of microtubule growth. Nature $312,237-242$.

Mitchison, TJ (1989). Polewards microtubule flux in the mitotic spindle: Evidence from photoactivation of fluorescence. J Cell Biol 109, 637-652.

Mitchison, TJ, Maddox, P, Gaetz, J, Groen, A, Shirasu, M, Desai, A, Salmon, ED, and Kapoor, TM (2005). Roles of polymerization dynamics, opposed motors, and a tensile element in governing the length of Xenopus extract meiotic spindles. Mol Biol Cell 16, 3064-3076.

Miyamoto, DT, Perlman, ZE, Burbank, KS, Groen, AC, and Mitchison, TJ (2004). The kinesin Eg5 drives poleward microtubule flux in Xenopus laevis egg extract spindles. J Cell Biol 167, 813-818.

Mogessie, B, Scheffler, K, and Schuh, M (2018). Assembly and Positioning of the Oocyte Meiotic Spindle. Annu Rev Cell Dev Biol 34, 381-403.

Moorhouse, KS, and Burgess, DR (2014). How to be at the right place at the right time: The importance of spindle positioning in embryos. Mol Reprod Dev 81, 884-895.

Nemergut, ME, Mizzen, CA, Stukenberg, T, Allis, CD, and Macara, IG (2001). Chromatin docking and exchange activity enhancement of RCC1 by histones H2A and H2B. Science 292, 1540-1543.

Nicklas, RB, and Gordon, GW (1985). The total length of spindle microtubules depends on the number of chromosomes present. J Cell Biol 100, 1-7.

Oh, D, Yu, C-H, and Needleman, DJ (2016). Spatial organization of the Ran pathway by microtubules in mitosis. Proc Natl Acad Sci 113, 8729-8734.

Ohi, R, Sapra, T, Howard, J, and Mitchison, TJ (2004). Differentiation of cytoplasmic and meiotic spindle assembly MCAK functions by Aurora B-dependent phosphorylation. Mol Biol Cell 15, 2895-2906.

Petry, S (2016). Mechanisms of Mitotic Spindle Assembly. Annu Rev Biochem 85, 659-683.

Petry, S, Groen, ACC, Ishihara, K, Mitchison, TJ, and Vale, RDD (2013). Branching Microtubule Nucleation in Xenopus Egg Extracts Mediated by Augmin and TPX2. Cell 152, $768-777$. 
Pietro, F, Echard, A, and Morin, X (2016). Regulation of mitotic spindle orientation: an integrated view. EMBO Rep 17, 1106-1130.

Reber, SB, Baumgart, J, Widlund, PO, Pozniakovsky, A, Howard, J, Hyman, AA, and Jülicher, F (2013). XMAP215 activity sets spindle length by controlling the total mass of spindle microtubules. Nat Cell Biol 15, 1-8.

Reis, R, Feijão, T, Gouveia, S, Pereira, AJ, Matos, I, Sampaio, P, Maiato, H, and Sunkel, CE (2009). Dynein and Mast/Orbit/CLASP have antagonistic roles in regulating kinetochoremicrotubule plus-end dynamics. J Cell Sci 122, 2543-2553.

Rieder, CL (1981). The structure of the cold-stable kinetochore fiber in metaphase PtK1 cells. Chromosoma 84, 145-158.

Rieder, CL, and Alexander, SP (1990). Kinetochores are transported poleward along a single astral microtubule during chromosome attachment to the spindle in newt lung cells. J Cell Biol 110, 81-95.

Rogers, GC, Rogers, SL, and Sharp, DJ (2005). Spindle microtubules in flux. J Cell Sci 118, $1105-1116$.

Sampath, SC, Ohi, R, Leismann, O, Salic, A, Pozniakovski, A, and Funabiki, H (2004). The chromosomal passenger complex is required for chromatin-induced microtubule stabilization and spindle assembly. Cell 118, 187-202.

Sauer, G, Körner, R, Hanisch, A, Ries, A, Nigg, EA, and Silljé, HHW (2005). Proteome Analysis of the Human Mitotic Spindle. Mol Cell Proteomics 4, 35-43.

Saunders, W, Lengyel, V, and Hoyt, M a (1997). Mitotic spindle function in Saccharomyces cerevisiae requires a balance between different types of kinesin-related motors. Mol Biol Cell $8,1025-1033$.

Savoian, MS, and Glover, DM (2010). Drosophila Klp67A binds prophase kinetochores to subsequently regulate congression and spindle length. J Cell Sci 123, 767-776.

Scholey, JM, Civelekoglu-Scholey, G, and Brust-Mascher, I (2016). Anaphase B. Biology (Basel) 5, 1-30.

Siller, KH, and Doe, CQ (2009). Spindle orientation during asymmetric cell division. Nat Cell Biol 11, 365-374.

Sluder, G (1976). Experimental manipulation of the amount of tubulin available for assembly into the spindle of dividing sea urchin eggs. J Cell Biol 70, 75-85.

Srayko, M, O’toole, ET, Hyman, A, and Müller-Reichert, T (2006). Katanin disrupts the microtubule lattice and increases polymer number in C. elegans meiosis. Curr Biol 16, 19441949. 
Stout, JR, Yount, AL, Powers, JA, LeBlanc, C, Ems-McClung, SC, and Walczak, C (2011). Kif18B interacts with EB1 and controls astral microtubule length during mitosis. Mol Biol Cell 22, 3070-3080.

Syrovatkina, V, Fu, C, and Tran, PT (2013). Antagonistic spindle motors and MAPs regulate metaphase spindle length and chromosome segregation. Curr Biol 23, 2423-2429.

Szollosi, D, Calarco, P, and Donahue, RP (1972). Absence of centrioles in the first and second meiotic spindles of mouse oocytes. J Cell Sci 11, 521-541.

Takagi, J, Sakamoto, R, Shiratsuchi, G, Maeda, YT, and Shimamoto, Y (2019). Mechanically Distinct Microtubule Arrays Determine the Length and Force Response of the Meiotic Spindle. Dev Cell 49, 267-278.e5.

Tao, L, Mogilner, A, Civelekoglu-Scholey, G, Wollman, R, Evans, J, Stahlberg, H, and Scholey, JM (2006). A Homotetrameric Kinesin-5, KLP61F, Bundles Microtubules and Antagonizes Ncd in Motility Assays. Curr Biol 16, 2293-2302.

Thawani, A, Stone, HA, Shaevitz, JW, and Petry, S (2019). Spatiotemporal organization of branched microtubule networks. Elife 8, 1-29.

Theurkauf, WE, and Hawley, RS (1992). Meiotic spindle assembly in Drosophila females: behavior of nonexchange chromosomes and the effects of mutations in the nod kinesin-like protein. J Cell Biol 116, 1167-1180.

Tokai-Nishizumi, N, Ohsugi, M, Suzuki, E, and Yamamoto, T (2005). The Chromokinesin Kid Is Required for Maintenance of Proper Metaphase Spindle Size. Mol Biol Cell 16, 54555463.

Uehara, R, Tsukada, Y, Kamasaki, T, Poser, I, Yoda, K, Gerlich, DW, and Goshima, G (2013). Aurora B and Kif2A control microtubule length for assembly of a functional central spindle during anaphase. J Cell Biol 202, 623-636.

Urbani, L, and Stearns, T (1999). The centrosome. Curr Biol 9, 315-317.

Vargas-Hurtado, D, Brault, J-BB, Piolot, T, Leconte, L, Da Silva, N, Pennetier, C, Baffet, A, Marthiens, V, and Basto, R (2019). Differences in Mitotic Spindle Architecture in Mammalian Neural Stem Cells Influence Mitotic Accuracy during Brain Development. Curr Biol 29, 2993-3005.e9.

Walczak, C, and Heald, R (2008). Mechanisms of Mitotic Spindle Assembly and Function. In: A Survey of Cell Biology, Elsevier Masson SAS, 111-158.

Walczak, C, Verma, S, and Mitchison, TJ (1997). XCTK2: a kinesin-related protein that promotes mitotic spindle assembly in Xenopus laevis egg extracts. J Cell Biol 136, 859-870. Walczak, C, Vernos, I, Mitchison, TJ, Karsenti, E, and Heald, R (1998). A model for the 
proposed roles of different microtubule-based motor proteins in establishing spindle bipolarity. Curr Biol 8, 903-913.

Walker, RA, O’Brien, ET, Pryer, NK, Soboeiro, MF, Voter, WA, Erickson, HP, and Salmon, ED (1988). Dynamic instability of individual microtubules analyzed by video light microscopy: rate constants and transition frequencies. J Cell Biol 107, 1437-1448.

Wang, H, Brust-Mascher, I, Cheerambathur, D, and Scholey, JM (2010). Coupling between microtubule sliding, plus-end growth and spindle length revealed by kinesin- 8 depletion. Cytoskeleton 67, 715-728.

Weaver, LN, Ems-McClung, SC, Chen, S-HHR, Yang, G, Shaw, SL, and Walczak, C (2015). The Ran-GTP gradient spatially regulates XCTK2 in the spindle. Curr Biol 25, 1509-1514.

Weaver, LN, Ems-McClung, SC, Stout, JR, Leblanc, C, Shaw, SL, Gardner, MK, and Walczak, C (2011). Kif18A uses a microtubule binding site in the tail for plus-end localization and spindle length regulation. Curr Biol 21, 1500-1506.

Wilbur, JD, and Heald, R (2013). Mitotic spindle scaling during Xenopus development by kif2a and importin $\alpha$. Elife 2, e00290.

Wilde, a, Lizarraga, SB, Zhang, L, Wiese, C, Gliksman, NR, Walczak, C, and Zheng, Y (2001). Ran stimulates spindle assembly by altering microtubule dynamics and the balance of motor activities. Nat Cell Biol 3, 221-227.

Wilde, A, and Zheng, Y (1999). Stimulation of microtubule aster formation and spindle assembly by the small GTPase Ran. Science 284, 1359-1362.

Wittmann, T, Boleti, H, Antony, C, Karsenti, E, and Vernos, I (1998). Localization of the Kinesin-like Protein Xklp2 to Spindle Poles Requires a Leucine Zipper, a Microtubuleassociated Protein, and Dynein. J Cell Biol 143, 673-685.

Wittmann, T, Wilm, M, Karsenti, E, and Vernos, I (2000). TPX2, A novel xenopus MAP involved in spindle pole organization. J Cell Biol 149, 1405-1418.

Wollman, R, Cytrynbaum, EN, Jones, JT, Meyer, T, Scholey, JM, and Mogilner, A (2005). Efficient chromosome capture requires a bias in the "search-and-capture" process during mitotic-spindle assembly. Curr Biol 15, 828-832.

Wühr, M, Chen, Y, Dumont, S, Groen, AC, Needleman, DJ, Salic, A, and Mitchison, TJ (2008). Evidence for an upper limit to mitotic spindle length. Curr Biol 18, 1256-1261.

Xu, Z, Vagnarelli, P, Ogawa, H, Samejima, K, and Earnshaw, WC (2010). Gradient of Increasing Aurora B Kinase Activity Is Required for Cells to Execute Mitosis. J Biol Chem 285, 40163-40170.

Yang, G, Cameron, LA, Maddox, PS, Salmon, ED, and Danuser, G (2008). Regional 
variation of microtubule flux reveals microtubule organization in the metaphase meiotic spindle. J Cell Biol 182, 631-639.

Young, S, Besson, S, and Welburn, JPI (2014). Length-dependent anisotropic scaling of spindle shape. Biol Open 3, 1217-1223.

Zhai, Y, Kronebusch, PJ, Simon, PM, and Borisy, GG (1996). Microtubule dynamics at the G2/M transition: Abrupt breakdown of cytoplasmic microtubules at nuclear envelope breakdown and implications for spindle morphogenesis. J Cell Biol 135, 201-214.

Zhang, MS, Furuta, M, Arnaoutov, A, and Dasso, M (2018). RCC1 regulates inner centromeric composition in a Ran-independent fashion. Cell Cycle 17, 739-748.

Zheng, Y, Wong, ML, Alberts, B, and Mitchison, T (1995). Nucleation of microtubule assembly by a $\gamma$-tubulin-containing ring complex. Nature $378,578-583$. 


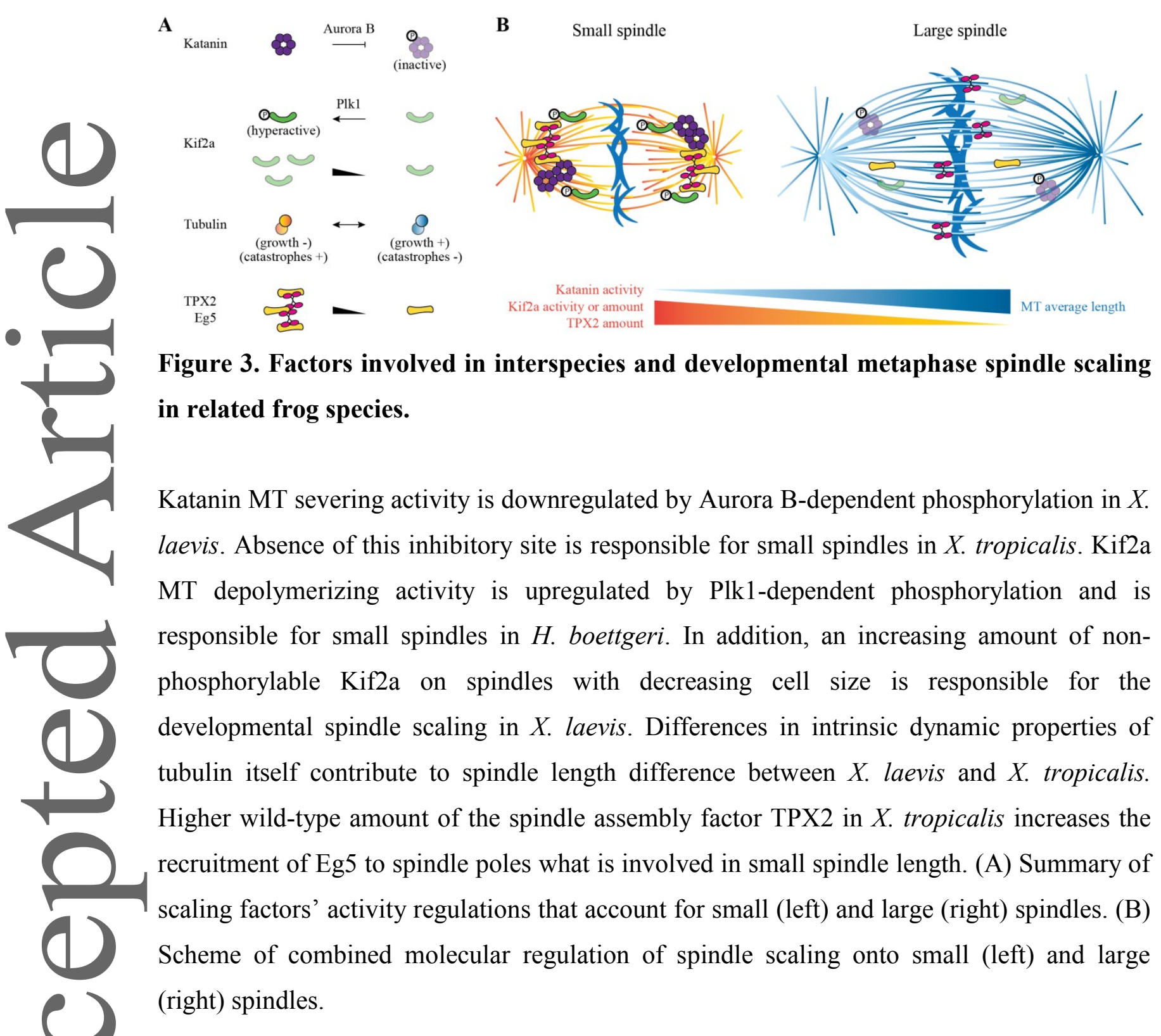




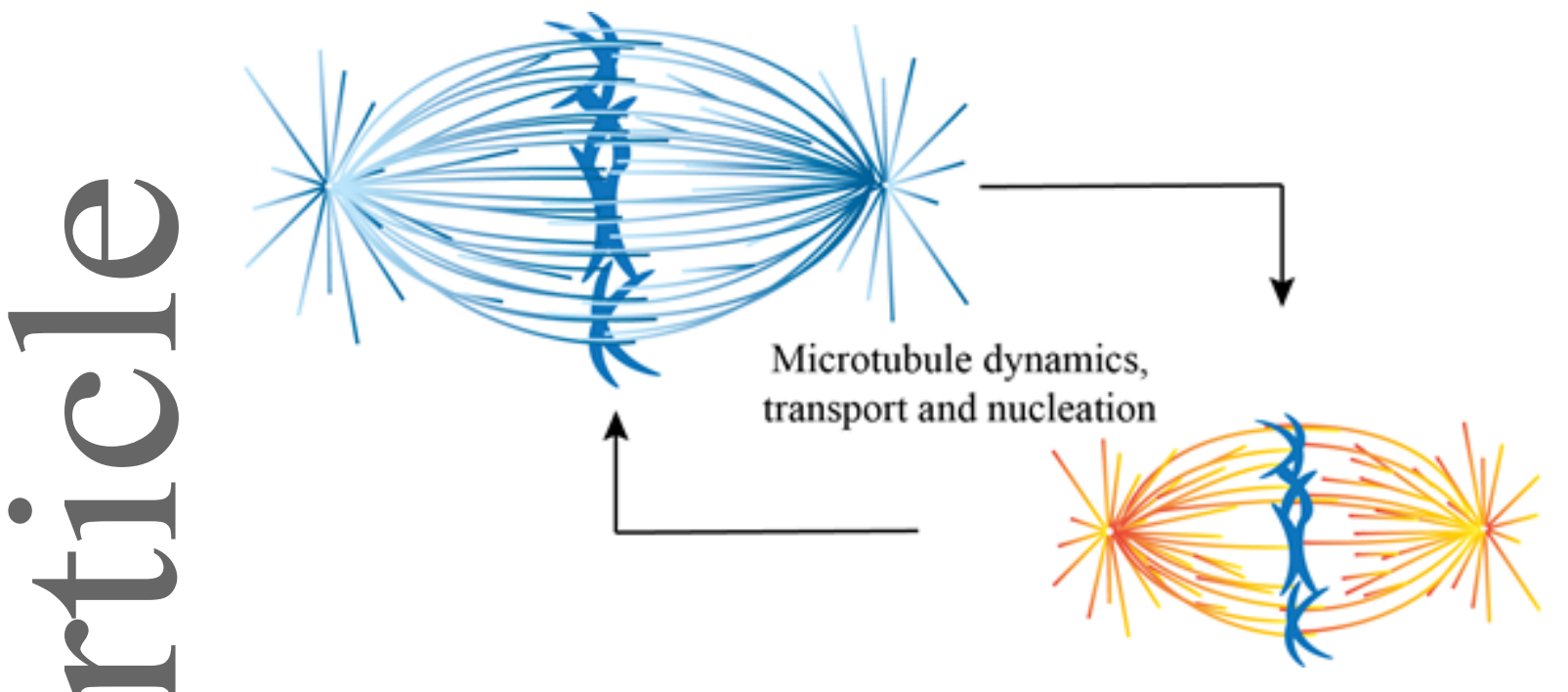

Outstanding discoveries were made over the past few years and changed our view of spindle assembly and organization. In particular, we now better understand how spindle size is regulated by physical constraints superimposed with molecular controls that spread from cell size sensing to the fine regulation of microtubule dynamics. Here, we review these latest studies and provide an overview of spindle architecture and size control mechanisms. 